\title{
EDITORIAL
}

\section{Does telehealthcare offer a patient-centred way forward for the community-based management of long-term respiratory disease?}

See linked articles by Smith et al. on pg 159 and Pinnock et al. on pg 233

Susannah McLean a and *Aziz Sheikh ${ }^{\text {b }}$

a Clinical Research Fellow

${ }^{b}$ Professor of Primary Care Research \& Development

Allergy \& Respiratory Research Group, Centre for Population Health Sciences: GP section, The University of Edinburgh, Scotland, UK

*Correspondence:

The University of Edinburgh

20 West Richmond Street

Edinburgh, Scotland, UK

EH8 9DX

E-mail: aziz.sheikh@ed.ac.uk
Caring for the millions of people with long-term respiratory conditions such as asthma and chronic obstructive pulmonary disease (COPD) is expensive and represents a major drain on healthcare resources internationally. The number and frailty of such patients is increasing rapidly, and this - coupled with changing expectations and the international concerns around spiralling healthcare budgets and economic downturn - is forcing governments and policymakers to look for novel cost-effective solutions to providing optimum care. There is considerable international interest that information technology (IT) will prove of pivotal importance in this quest, and in particular the novel ways of delivering care remotely through telehealthcare interventions.

Respiratory conditions now pose an enormous disease burden worldwide. For example, the Global Initiative for Asthma (GINA) estimates that 300 million people suffer from asthma, and in some developed English-speaking countries the prevalence of asthma is as high as $30 \%$ in age groups under $40 .{ }^{1}$ Similarly, in the UK, over 27,000 people died from COPD in 2004, and cases of COPD take up more than 1 million hospital bed days a year. In 2004, respiratory disease cost the UK $£ 6.6$ billion - $f 3$ billion in NHS care costs, $£ 1.9$ billion in mortality costs and $\mathrm{f} 1.7$ billion in morbidity costs. ${ }^{2}$

The term "telehealthcare" refers to the electronic transfer of patient-generated data to a remotely-located healthcare professional providing personalised patient care. Telehealthcare thus includes, but is not restricted to, "telemedicine", which involves synchronous doctor-patient teleconsulting either by phone or video-linkage. Also embraced within telehealthcare are a number of other means of synchronous and asynchronous consulting, such as instant messaging, short message service (SMS), Skype, e-mail, text messaging and web-based consulting.

Finkelstein and Friedman ${ }^{3}$ have detailed a range of potential benefits to patients associated with telehealthcare interventions (see Box 1 for a discussion of these in the context of respiratory disease). The proactive use of such technologies can, however, involve costly re-organisation of services, the need to develop new care pathways, and training costs for staff. Policy makers hope (and increasingly believe) that these high start-up costs will be offset by lower running costs in the medium- to longer-term.

The potential risks of telehealthcare initiatives also need to be considered. At the population level, there is the risk of exacerbating the "digital divide" - the inequalities between people who have the digital technology and those (often older and/or socioeconomically deprived) people who do not. There have been efforts to address this issue generically - for example, Gordon Brown's announcement at the recent UK Labour Party Conference that $\mathrm{f} 300$ million would be invested to pay for free broadband internet connection for all children between the age of seven and 19 in low income and jobless families. However, this will not reach everyone in need, and older people, in particular, may lose out.

There is also concern that vulnerable populations such as frail older people will struggle to use the technology due to its complexity. Thus, there needs to be a relentless drive to make telehealthcare as user-friendly as possible. This accessibility does, however, need to be

\footnotetext{
Acknowledgements

AS gratefully acknowledges the support received through grants from the NHS Connecting for Health Evaluation Programme, Asthma UK, the Chief Scientist's Office of the Scottish Government and the MRC Translational Medicine Methodology Trial Hub.
} 
counter-balanced by the need to protect against breaches of confidentiality - a risk that still persists even with widespread use of password and/or biometric technology and encryption software.

On the technical side, inter-operability standards for systems need to be set centrally by government to ensure that systems produced by independent companies can communicate seamlessly with each other. This need for interoperability also produces a tension with confidentiality as a wider range of companies will need to have access to the encrypting codes.

Telehealthcare is increasingly being combined with decision support functionality, this tailored advice aimed at either the patient, healthcare provider, or both. Whilst undoubtedly potentially helpful, this additional functionality is crucially dependent on carefully developed algorithms of care; the risks associated with poorly developed algorithms are substantial.

The set-up costs of these systems and the investment in the technology to address these risks needs to be justified by their benefits to patients and their carers. Of concern, then, is that a recent Cochrane systematic review of generic teleconsultations compared with face-to-face consultations found a paucity of high quality evidence to inform clinical practice. From the available trials the reviewers concluded that there is as yet little evidence of clinical benefit, variable and inconclusive results for other outcomes such as psychological outcomes, and there were no data on costeffectiveness. ${ }^{4}$ Similarly, another systematic review of studies of patient satisfaction with telehealthcare raised more questions than it answered; these included, amongst others, the need to understand the impact of these novel modes of consulting on the cherished doctor-patient relationship. ${ }^{5}$ Answers to such fundamental questions about benefits and risks clearly need to be considered before rushing head-long into investment in heavilymarketed telehealthcare solutions.

In conclusion, the evidence base for these technologies is, as Smith and colleagues demonstrate in this issue, ${ }^{6}$ at present very weak and there are many possible risks that need to be carefully considered. Effectiveness has not yet been proven, and costeffectiveness has been barely studied. ${ }^{6,7,8}$ That said, it is undeniable that telehealthcare offers very significant opportunities to transform healthcare delivery, and so the lack of evidence must not be a bar to continuing careful investment in this area. In view of this tension, and the fact that there are strong policy drivers to such developments, one way forward is to work towards better synchronisation and implementation of telehealthcare technologies with real-time independent and rigorous evaluations. Our experiences suggest that this is far from straight-forward, but with methodological creativity this can be achieved. This is, for example, being demonstrated in the trial protocol by Pinnock and colleagues reported in this issue, which seeks to establish the effectiveness and cost-effectiveness of a new telemetry service established for people with COPD in Lothian, Scotland. ${ }^{9}$ Ongoing Cochrane systematic reviews will furthermore provide greater clarity on the role of telehealthcare in respiratory disorders in due course. ${ }^{10,11}$
Box 1: Potential benefits associated with telehealthcare interventions, adapted from Finkelstein and Friedman ${ }^{3}$

- $\quad$ Allows patients to be cared for in their preferred location i.e. typically at home.

- Videophone or web-based clinical consultations e.g. asthma or COPD annual reviews can replace routine visits such as annual reviews.

- $\quad$ Proactive education and support e.g. through web forums, may facilitate self-management techniques and help prevent exacerbations.

- $\quad$ Use of telemonitoing of respiratory measures such as peak expiratory flow and spirometry can allow earlier detection of disease exacerbations, thereby facilitating timely management and support.

- $\quad$ Greater opportunities for continuity of care.

- $\quad$ Reduced costs to patients resulting from savings in time off work, and obviating transport and parking costs.

\section{Conflict of interest declaration}

AS is an Assistant Editor of the PCRJ, but was not involved in the editorial review of, nor the decision to publish, this article.

\section{References}

1. GINA, Masoli M, Fabia D, Holt S, Beasley R, Global Burden of Asthma. The Global Initiative for Asthma 12/22/03.

2. BOLD The Burden of Lung Disease, 2nd Edition. A Statistics Report from the British Thoracic Society 2006.

3. Finkelstein J, Friedman RH. Potential Role of Telecommunication Technologies in the Management of Chronic Health Conditions. Disease Management Health Outcomes 2000;2:57-63. http://dx.doi.org/10.2165/00115677200008020-00001

4. Currell R, Urquhart C, Wainwright $P$, Lewis R. Telemedicine versus face to face patient care: effects on professional practice and health care outcomes. Cochrane Database of Systematic Reviews 2000;(2): Art . No.:CD002098. http://dx.doi.org/10.1002/14651858.

5. Mair F, Whitten P. Systematic review of studies of patient satisfaction with telemedicine. BMJ 2000;320:1517-20. http://dx.doi.org/10.1136/ bmj.320.7248.1517

6. Smith SM, Elkin SL, Partridge MP. Technology and its role in respiratory care. Prim Care Resp J 2009;18(3):159-64. http://dx.doi.org/ 10.4104/pcrj.2009.00038

7. Whitten PS, Mair FS, Haycox A, May CL, Williams TL, Hellmich S. Systematic review of cost effectiveness studies of telemedicine interventions. BMJ 2002;324:1434-7. http://dx.doi.org/10.1136/bmj.324.7351.1434

8. Car J, Anandan C, Black A, Cresswell K, Pagliari C, McKinstry B, Sheikh A. Impact of eHealth on the Quality \& Safety of Healthcare - A systematic overview and synthesis of the literature. Report for the NHS Connecting for Health Evaluation Programme, 2008. Available from: http://www.pcpoh. bham.ac.uk/publichealth/cfhep/documents/NHS_CFHEP_001_Final_Report.pdf (last accessed 7 November 2008)

9. Pinnock $\mathrm{H}$, Hanley J, Lewis $\mathrm{S}$, et al. Impact of a telemetric COPD monitoring service: RCT with economic evaluation and nested qualitative study. Prim Care Resp J 2009;18(3):233-5. http://dx.doi.org/10.4104/pcrj.2009.00040

10. McLean S, Liu J, Pagliari C, Car J, Sheikh A. Telehealthcare for asthma (Protocol). Cochrane Database of Systematic Reviews 2009, Issue 2. Art. No.: CD007717. http://dx.doi.org/10.1002/14651858.CD007717.

11. McLean S, Liu J, Pagliari C, Car J, Sheikh A. Telehealthcare for chronic obstructive pulmonary disease (Protocol). Cochrane Database of Systematic Reviews 2009, Issue 2. Art. No.: CD007718. http://dx.doi.org/10.1002/14651858.CD007718. 